\title{
CONTROLE DA ANTRACNOSE E QUALIDADE DE MANGAS (Mangifera indica L.) cV. VAN DYKE, APÓS TRATAMENTO HIDROTÉRMICO E QUÍMICO
}

\author{
Anthracnose control and mango quality (Mangifera indica L.) cv. van dyke after \\ hydrothermal and chemical treatment
}

\author{
Mário Sérgio Carvalho Dias ${ }^{1}$, Luciana Costa Lima², Marcos Venícios de Castro ${ }^{3}$, \\ Pedro Martins Ribeiro Júnior ${ }^{4}$, Enilson de Barros Silva ${ }^{5}$
}

\section{RESUMO}

A antracnose causa severos danos à casca da manga. Por isso, há a necessidade de se fazer o tratamento fitossanitário pós-colheita dos frutos, utilizando a imersão em calda de fungicidas, a qual, associada ao tratamento hidrotérmico, tem resultado em melhores benefícios. Com o presente trabalho objetivou-se estudar o controle da antracnose e qualidade pós-colheita de mangas (Mangifera indica L.), cv. Van Dyke, após tratamento hidrotérmico associado ao químico mediante as análises física, físicoquímicas, químicas e fitopatológica. As mangas, provenientes de pomar comercial do município de Janaúba - MG, da safra 2000, foram colhidas em estádio de maturação comercial. Após seleção de acordo com a uniformidade de cor, tamanho e ausência de injúrias mecânicas e fisiológicas, as mesmas foram transportadas em caixas plásticas para a EPAMIG - CTNM - Nova Porteirinha - MG, Laboratório de Pós-colheita de frutos, onde as amostras foram submetidas ao tratamento hidrotérmico $\left(55^{\circ} \mathrm{C}\right.$ por 5minutos) e banho frio em soluções contendo os fungicidas Thiabendazole (Tecto $400 \mathrm{~mL} / 100 \mathrm{~L}$ ), Prochloraz (Sportak 110 mL/100 L) e Imazalil (Magnate $200 \mathrm{~mL} / 100 \mathrm{~L}$ ). Após secagem ao ar, os frutos foram acondicionados em bandejas plásticas e armazenados à temperatura ambiente $\left(25 \pm 2{ }^{\circ} \mathrm{C}\right.$ e UR $\left.70 \%\right)$, por um período de 12 dias e avaliados quanto às características intrínsecas de qualidade a cada 4 dias. O experimento foi conduzido em delineamento inteiramente casualizado, com 8 tratamentos, 4 repetições e unidade experimental composta de 4 frutos. As variações de $\mathrm{pH}$, sólidos solúveis totais, acidez total titulável e açúcares solúveis totais não comprometeram as características organolépticas de mangas cv. Van Dyke armazenadas sob temperatura ambiente $\left(25 \pm 2^{\circ} \mathrm{C}\right.$ e UR $\left.70 \%\right)$ até os 8 dias de armazenamento. A associação do tratamento hidrotérmico com o químico foi eficiente no controle da antracnose dos frutos por até 12 dias de armazenamento. O fungicida Prochloraz (Sportak 110 $\mathrm{mL} / 100 \mathrm{~L}$ ), associado ao tratamento hidrotérmico, inibiu totalmente o aparecimento de sintomas de antracnose.

Termos para indexação: conservação e fungicida.

\begin{abstract}
Anthracnose causes severe damage to the mango skin. Therefore, there is need to do the post-harvest phytosanitary treatment of the fruits through soaking in fungicide solution, which associated with the hydrothermal treatment has resulted into improved benefits. The present work has aimed at studying anthracnose control and post-harvest quality of mangoes (Mangifera indica L.), cv. Van Dyke after hydrothermal treatment associated with chemical through the physical, physicochemical, chemical and phytopathologic analyses. The mangoes coming from the commercial orchard of the town of Janaúba-MG, of the 2000 crop, were harvested at the commercial maturation stage. After selection according to color uniformity, size and absence of mechanic and physiologic injuries, they were shipped in plastic boxes to the EPAMIG - CTNM - Nova Porteirinha - MG, fruit post-harvest laboratory, where the samples were submitted to the hydrothermal treatment in cold bath in solution containing the fungicides Thiabendazole (Tecto $400 \mathrm{~mL} / 100 \mathrm{~L}$ ), Proclaraz (Sportak 10L/100L) and Imazalil (Magnate 200mL/100L). After air-drying, the fruits were packed in plastic trays and stored at room temperature $\left(25 \pm 2^{\circ} \mathrm{C}\right.$ and $\left.\mathrm{RH} 70 \%\right)$ for a 12 -day period and evaluated as to the intrinsic quality characteristics every 4 days. The experiment was conducted a completely randomized with 8 treatments, 4 replicates and experimental unit consisting of 4 fruits. The variations of $\mathrm{pH}$, total soluble solids, total titrable acidity and total soluble sugars have not endangered the organoleptic characteristics of mangoes cv. Van Dyke stored under room temperature $\left(25 \pm 2^{\circ} \mathrm{C}\right.$ and $\left.\mathrm{RH} 70 \%\right)$ till 8 days' storage. The association of the hydrothermal treatment with the chemical was efficient in fruit anthracnose control for till 12 days' storage. The fungicide Prochloraz (Sportak 110 mL/100L), associated with the hydrothermal treatment, completely inhibited the appearance of anthracnose symptoms.
\end{abstract}

Index Terms: conservation and fungicide.

(Recebido para publicação em 30 de abril de 2004 e aprovado em 20 de Janeiro de 2005)

1. Engenheiro Agrônomo, D.Sc., Pesquisador da EPAMIG - CTNM - Nova Porteirinha, MG - Caixa Postal 12 - 39.440-000 - diasmsc@nortecnet.com.br

2. Engenheiro Agrônomo, M.Sc., Doutoranda em Horticultura na FCA da UNESP de Botucatu - Botucatu,SP - limalc@fca.unesp.br

3. Biólogo, M.Sc., Professor Adjunto da Univale - Governador Valadares,MG -marcoscastro@hotmail.com

4. Engenheiro Agrônomo, Aluno de Mestrado em Fitopatologia da Universidade Federal de Lavras/UFLA - Caixa Postal 3037 - $37.200-000$ Lavras,MG - pedromartinsjr@bol.com.br

5. Engenheiro Agrônomo, D.Sc., Professor Adjunto da FAFEOD - Diamantina, MG - benilson@hotmail.com 


\section{INTRODUÇÃO}

A mangueira é uma espécie originária da Índia, estando atualmente distribuída por praticamente todas as regiões tropicais do mundo, e inclusive sendo cultivada comercialmente em algumas regiões de clima subtropical (KIST et al., 1996). O interesse pela cultura deve-se a excelência de seus frutos que, além de seu sabor exótico, são ricos em vitaminas e sais minerais, e em relação a vitamina $\mathrm{A}$, a manga se constitui numa das frutas mais ricas.

A produção comercial no Brasil está localizada principalmente nas regiões Sudeste e Nordeste do País, onde as temperaturas permanecem elevadas quase $o$ ano inteiro. Os principais estados produtores são: São Paulo, Minas Gerais, Paraíba, Piauí, Ceará, Bahia e Pernambuco e as principais cultivares utilizadas são: Tommy Atkins, Haden, Keitt, Van Dyke e Rosa (SÃO JOSÉ, 1996).

A aparência da manga é o fator mais importante do sucesso na sua comercialização. A casca tem que ser perfeita até chegar ao consumidor final, o que é muito difícil, já que a manga é um fruto frágil e pode estar infectada por Colletotrichum gloeosporioides Penz, fungo responsável pela antracnose (GAYET, 1994).

Alguns autores recomendam, que o controle póscolheita da doença deva ser feito pela da imersão dos frutos em solução de Thiabendazole a $0,2 \%$ ou Benomyl a 0,1\% mais espalhante adesivo, por 5 minutos. Cunha et al. (1994) recomendam que o tratamento químico da antracnose da mangueira deva ser feito mediante pulverizações com fungicidas à base de cobre, Mancozeb e Benomyl. Para uma maior eficácia, recomenda-se a associação com o tratamento hidrotérmico.

Conduziu-se este trabalho com o objetivo de estudar o controle da antracnose e qualidade pós-colheita de mangas cv. Van Dyke após tratamento hidrotérmico associado ao químico e armazenamento em temperatura ambiente ( $25 \pm 2^{\circ} \mathrm{C}$ e UR $70 \%$ ) por um período de 12 dias.

\section{MATERIAL E MÉTODOS}

Mangas, provenientes de pomar comercial do município de Janaúba - MG, da safra 2000, foram colhidas em estádio de maturação comercial. Após seleção de acordo com a uniformidade de cor, tamanho e ausência de injúrias mecânicas e fisiológicas, as mesmas foram transportadas em caixas plásticas para a Empresa de Pesquisa Agropecuária do Norte de Minas Gerais EPAMIG - CTNM - Nova Porteirinha - MG, Laboratório de Pós-colheita de Frutos, onde as amostras foram submetidas a tratamento hidrotérmico $\left(55^{\circ} \mathrm{C}\right.$ por $5 \mathrm{mi}-$ nutos) e banho frio em soluções contendo os seguintes fungicidas: T1 - banho frio em solução com Thiabendazole (Tecto $400 \mathrm{~mL} / 100 \mathrm{~L}$ ); $\mathbf{T 2}$ - banho frio em solução com Prochloraz (Sportak 110 mL/100 L; T3 - banho frio em solução com Imazalil (Magnate 200 mL/100 L); T4 - banho quente em solução com Thiabendazole (Tecto $400 \mathrm{~mL} / 100 \mathrm{~L}$ a $55^{\circ} \mathrm{C}$ ); $\mathbf{T 5}$ - banho quente em solução com Prochloraz (Sportak 110 $\mathrm{mL} / 100 \mathrm{~L}$ a $55^{\circ} \mathrm{C}$ ); $\mathbf{T 6}$ - banho quente em solução com Imazalil (Magnate $200 \mathrm{~mL} / 100 \mathrm{~L}$ a $55^{\circ} \mathrm{C}$ ); $\mathbf{T} 7$ - Testemunha a $55^{\circ} \mathrm{C}$; $\mathbf{T 8}$ - Testemunha absoluta. Após secagem ao ar, os frutos foram acondicionados em bandejas plásticas e armazenados à temperatura ambiente $\left(25 \pm 2^{\circ} \mathrm{C}\right.$ e UR $\left.70 \%\right)$, por um período de 12 dias e avaliados quanto às características intrínsecas de qualidade a cada 4 dias.

O experimento foi conduzido em delineamento inteiramente casualizado, com 8 tratamentos, 4 repetições e unidade experimental composta de 4 frutos.

Durante o armazenamento, procedeu-se à caracterização física dos frutos quanto ao peso (g) e à perda de massa (\%), por pesagem individual dos frutos. As análises físico-químicas e químicas foram realizadas após homogeneização da polpa e constaram das seguintes variáveis: $\mathrm{pH}$, medido por potenciometria (AOAC, 1990); sólidos solúveis totais obtidos por refratometria, expressando-se os resultados em ${ }^{\circ}$ Brix (AOAC, 1990); acidez total titulável, por titulação com hidróxido de sódio $0,1 \mathrm{~N}$ e fenolftaleína como indicador expressando-se os resultados em Mol L ${ }^{-1}$ (IAL, 1985); açucares solúveis totais (\% de glicose, frutose e sacarose) dosados pela técnica de Somogyi adaptada por Nelson (1944).

Quanto à análise fitopatológica, foram atribuídas notas de 0 a 5 de acordo com o grau de infecção, sendo: 0 = ausência de infecção; $1=$ até $10 \%$ de infecção; 2 = 10,1 a 20\% de infecção; $3=20,1$ a 30\% de infecção; 4 $=30,1$ a $40 \%$ de infecção e 5 = acima de $40 \%$ de infecção. Determinado o grau de infecção, os dados da escala numérica foram transformados em porcentagem de infecção por parcela de acordo com a fórmula a seguir :

\footnotetext{
Porcentagem de infecção $=$

$\frac{=(\mathrm{nx} 0)+(\mathrm{nx} 1)+(\mathrm{nx} 2)+(\mathrm{nx} 3)+(\mathrm{nx} 4)+(\mathrm{nx} 5) \times 100}{5 \times \mathrm{n} \text { total }}$

$\mathrm{n}=$ número de frutos avaliados por parcela.

Os dados foram submetidos à análise de variância, e as médias foram comparadas pelo Teste de Tukey ao nível de $1 \%$ de probabilidade, segundo Gomes (1985).
} 


\section{RESULTADOS E DISCUSSÃO}

Pelos resultados da Tabela 1, a variável perda de massa foi influenciada pelo tempo de armazenamento $(\mathrm{P}<0,01)$. Os frutos perderam $6,5 \%$ da sua massa quando armazenados por 12 dias à temperatura ambiente $\left(25 \pm 2^{\circ} \mathrm{C}\right.$ e UR $\left.70 \%\right)$. Resultados semelhantes foram obtidos por Bliska (1996), que verificou que a manga armazenada de 8 a $10^{\circ} \mathrm{C}$ e UR $90 \%$ perde $6,5 \%$ do seu peso no período de 3 a 4 semanas.

A média de peso dos frutos obtida neste trabalho foi de $347 \mathrm{~g}$ (Tabela 1), valor esse, também obtido por Siqueira et al. (1988) e Souza et al. (1984), que trabalharam com frutos de diversas cultivares e encontraram pesos que variaram entre $132 \mathrm{a} 602 \mathrm{~g}$. O valor encontrado está dentro da faixa exigida pelo mercado europeu, que admite frutas com peso de 250 até $750 \mathrm{~g}$, dando preferência às mangas com $300 \mathrm{a}$ 400 g. Já o consumidor americano aceita frutas de 250 até 650 g, porém, prefere a manga com peso em torno de $400 \mathrm{~g}$ (BLEINROTH, 1994).
A variável $\mathrm{pH}$ foi influenciada pela ação conjunta do tipo de fungicida e do banho aplicado (Tabela 2). Também houve interação entre e tipo de banho aplicado e tempo de armazenamento $(\mathrm{P}<0,01)$, conforme verifica-se pela Tabela 3 .

Os frutos testemunha a $55^{\circ} \mathrm{C}$ e imersos em solução de Imazalil (Magnate $200 \mathrm{~mL} / 100 \mathrm{~L}$ em tratamento hidrotérmico), apresentaram menores valores de $\mathrm{pH}$, conforme verifica-se na Tabela 2. Porém esses valores foram pouco inferiores, demonstrando dessa forma que a imersão em fungicidas não causa grandes variações no $\mathrm{pH}$.

Siqueira et al. (1988) citam que para a utilização industrial, um pH inferior a 4,3 é desejável, pois confere ao produto maior resistência à contaminações microbianas. Além disso, quanto menor o $\mathrm{pH}$ natural dos frutos, menor será a necessidade de adição artificial de ácidos para o controle de contaminações, reduzindo dessa forma o custo de industrialização.

$\mathrm{O}$ pH também sofreu modificações ao longo do armazenamento e aumentou durante o período experimental (Tabela 3).

TABELA 1 - Valores médios de peso (g) e perda de massa (\%) de mangas cv. Van Dyke, armazenadas a temperatura ambiente $\left(25 \pm 2^{\circ} \mathrm{C}\right.$ e UR 70\%), por um período de 12 dias. Safra 2000, Janaúba - MG, ano 2000.

\begin{tabular}{ccc}
\hline Armazenamento (dias) & Peso (g) & Perda de massa (\%) \\
\hline 0 & $356 \mathrm{a}$ & 0,0 \\
4 & $355 \mathrm{a}$ & 0,3 \\
8 & $345 \mathrm{ab}$ & 3,1 \\
12 & $333 \mathrm{~b}$ & 6,5 \\
\hline
\end{tabular}

Médias seguidas de mesma letra, não diferem entre si, ao nível de 1\% de significância pelo Teste de Tukey.

TABELA 2 - Valores médios de pH de mangas cv. Van Dyke, após tratamento hidrotérmico e químico e armazenamento em temperatura ambiente $\left(25 \pm 2^{\circ} \mathrm{C}\right.$ e UR $\left.70 \%\right)$, por um período de 12 dias. Safra 2000, Janaúba - MG, ano 2000.

\begin{tabular}{lcc}
\hline \multicolumn{1}{c}{ Fungicidas } & \multicolumn{2}{c}{ pH } \\
\cline { 2 - 3 } & Banho Frio & Banho Quente \\
\hline Thiabendazole (Tecto $400 \mathrm{~mL} / 100 \mathrm{~L})$ & $3,83 \mathrm{aA}$ & $3,84 \mathrm{aA}$ \\
Prochloraz (Sportak $110 \mathrm{~mL} / 100 \mathrm{~L})$ & $3,84 \mathrm{aA}$ & $3,74 \mathrm{aA}$ \\
Imazalil (Magnate $200 \mathrm{~mL} / 100 \mathrm{~L})$ & $3,94 \mathrm{aA}$ & $3,70 \mathrm{bB}$ \\
Testemunha & $3,78 \mathrm{aA}$ & $3,61 \mathrm{bB}$ \\
\hline
\end{tabular}

Médias seguidas de mesma letra, minúscula na horizontal e maiúscula na vertical, não diferem entre si ao nível de 1\% de significância pelo Teste de Tukey. 
TABELA 3 - Valores médios de pH de mangas cv. Van Dyke, após tratamento hidrotérmico e armazenamento em temperatura ambiente $\left(25 \pm 2^{\circ} \mathrm{C}\right.$ e UR 70\%), por um período de 12 dias. Safra 2000, Janaúba - MG, ano 2000.

\begin{tabular}{ccc}
\hline Tempo de Armazenamento (dias) & \multicolumn{2}{c}{$\mathbf{p H}$} \\
\cline { 2 - 3 } & Banho Frio & Banho Quente \\
\hline 0 & $3,01 \mathrm{aD}$ & $3,01 \mathrm{aD}$ \\
4 & $3,41 \mathrm{aC}$ & $3,33 \mathrm{aC}$ \\
8 & $4,33 \mathrm{aB}$ & $3,98 \mathrm{bB}$ \\
12 & $4,64 \mathrm{aA}$ & $4,56 \mathrm{aA}$ \\
\hline
\end{tabular}

Médias seguidas de mesma letra, minúscula na horizontal e maiúscula na vertical, não diferem entre si ao nível de 1\% de significância pelo Teste de Tukey.

O pH médio apresentado pelos frutos foi de 3,78, valor esse dentro da faixa ideal de 3,70 a 4,65 para a industrialização citada por Siqueira et al. (1988).

O teor de sólidos solúveis totais (SST) foi influenciado pelo tipo de fungicida utilizado e tempo de armazenamento $(\mathrm{P}<0,01)$. Frutos-testemunha a $55^{\circ} \mathrm{C}$ e imersos em Prochloraz (Sportak $110 \mathrm{~mL} / 100 \mathrm{~L}$ ) após tratamento hidrotérmico, apresentaram menor teor de SST conforme mostra a Tabela 4.

No decorrer do período experimental, observou-se uma tendência de aumento no teor de SST até os 8 dias de armazenamento e um declínio após esse período (Tabela 5).

Durante o estádio de maturação, o amido da polpa se transforma em açúcares, elevando dessa forma os teores de SST. As perdas transpiracionais também levam a uma maior concentração no teor de SST. A queda observada aos 12 dias de armazenamento (Tabela 5) pode ser explicada pela utilização da glicose produzida na quebra do amido nos processos respiratórios do fruto.

O teor médio de SST para os frutos analisados foi de 10,57\% (Tabela 5), valor esse inferior ao observado por Siqueira et al. (1988), que trabalhando com diversas cultivares encontrou valores que oscilaram de 11,40 a 21,56\%.

De acordo com Rodrigues (1977), um elevado teor de SST nos frutos, além de satisfazer a preferência do consumidor brasileiro, é muito importante quando o produto é industrializado, pois reduz a necessidade de adição de açúcar. Já para o mercado externo, segundo Souza et al. (1984), a preferência é por frutas com menor teor de SST, em relação ao mercado brasileiro.

A acidez total titulável (ATT) foi influenciada pelos fungicidas durante o armazenamento $(\mathrm{P}<0,01)$. Em todos os tratamentos a tendência foi de aumento na ATT até os 4 dias, com redução até o final do armazenamento (Tabela 6).

O teor de ácidos orgânicos, com poucas exceções, diminui com a maturação, em decorrência do processo respiratório ou de sua conversão em açúcares (CHITARRA \& CHITARRA, 1990). Teores de ATT obtidos para as cultivares Ruby, Palmer e Tommy Atkins por Maia et al. (1986), estão dentro do intervalo de 0,14 a $0,76 \mathrm{Mol} \mathrm{L}^{-1}$, teor esse bastante inferior ao do presente trabalho que apresentou em média 1,60 $\mathrm{Mol} \mathrm{L}^{-1}$.

Segundo Souza et al. (1984), frutos com menor acidez são preferidos pelo consumidor brasileiro, enquanto os frutos com maior acidez são indicados para satisfazer as exigências do consumidor estrangeiro, bem como para a sua industrialização.

Conforme os dados da Tabela 7, os açúcares solúveis totais (AST), sofreram influência de todas as variáveis estudadas $(\mathrm{P}<0,01)$. A tendência foi de aumento até os oito dias de armazenamento sofrendo declínio após esse período.

Segundo Chitarra \& Chitarra (1990), o teor de açúcares normalmente aumenta após a colheita e também durante o armazenamento por curtos períodos, por meio de processos de biossíntese ou pela degradação de polissacarídeos, entretanto, após o armazenamento prolongado, todos os açúcares decrescem. Medina (1996), também verificou que os teores de açúcares solúveis totais aumentam gradualmente, seguindo-se uma ligeira queda durante a maturação.

Quanto à avaliação fitopatológica, verificou-se que frutos não tratados com fungicida apresentaram alta incidência de antracnose. O tratamento hidrotérmico e químico diminuiu a incidência da infecção (Tabela 8).

O melhor resultado obtido foi após a associação de Prochloraz (Sportak $110 \mathrm{~mL} / 100 \mathrm{~L}$ em tratamento hidrotérmico), em que não foi verificado sintomas de antracnose nos frutos.

Sampaio (1981) verificou que mangas das cvs. Bourbon e Haden, após tratamento térmico e químico apresentam perdas na comercialização devido à antracnose, entretanto, as perdas são maiores nos frutos não tratados. 
TABELA 4 - Valores médios de sólidos solúveis totais ( ${ }^{\circ}$ Brix) de mangas cv. Van Dyke, após tratamento hidrotérmico e químico e armazenamento em temperatura ambiente $\left(25 \pm 2^{\circ} \mathrm{C}\right.$ e UR $\left.70 \%\right)$, por um período de 12 dias. Safra 2000, Janaúba - MG, ano 2000.

\begin{tabular}{lcc}
\hline \multicolumn{1}{c}{ Fungicidas } & \multicolumn{2}{c}{ Sólidos Solúveis Totais (\%) } \\
\cline { 2 - 3 } & Banho Frio & Banho Quente \\
\hline Thiabendazole (Tecto $400 \mathrm{~mL} / 100 \mathrm{~L})$ & $10,98 \mathrm{a}$ & $10,25 \mathrm{a}$ \\
Prochloraz (Sportak $110 \mathrm{~mL} / 100 \mathrm{~L})$ & $11,09 \mathrm{a}$ & $9,80 \mathrm{~b}$ \\
Imazalil (Magnate $200 \mathrm{~mL} / 100 \mathrm{~L})$ & $10,51 \mathrm{a}$ & $11,00 \mathrm{a}$ \\
Testemunha & $11,05 \mathrm{a}$ & $9,89 \mathrm{~b}$ \\
\hline
\end{tabular}

Médias seguidas de mesma letra, não diferem entre si, ao nível de 1\% de significância pelo Teste de Tukey.

TABELA 5 - Valores médios de sólidos solúveis totais ( ${ }^{\circ}$ Brix) de mangas cv. Van Dyke, armazenadas em temperatura ambiente $\left(25 \pm 2^{\circ} \mathrm{C}\right.$ e UR 70\%), por um período de 12 dias. Safra 2000, Janaúba - MG, ano 2000.

\begin{tabular}{cc}
\hline Armazenamento (dias) & Sólidos Solúveis Totais (\%) \\
\hline 0 & $5,53 \mathrm{~d}$ \\
4 & $10,04 \mathrm{c}$ \\
8 & $14,37 \mathrm{a}$ \\
12 & $12,35 \mathrm{~b}$ \\
\hline
\end{tabular}

Médias seguidas de mesma letra, não diferem entre si, ao nível de 1\% de significância pelo Teste de Tukey.

TABELA 6 - Valores médios de acidez total titulável $\left(\mathrm{Mol} \mathrm{L}^{-1}\right)$ de mangas cv. Van Dyke, após imersão em fungicidas e armazenamento em temperatura ambiente $\left(25 \pm 2^{\circ} \mathrm{C}\right.$ e UR $\left.70 \%\right)$, por um período de 12 dias. Safra 2000, Janaúba - MG, ano 2000.

\begin{tabular}{lcccc}
\hline \multirow{2}{*}{ Fungicidas } & \multicolumn{3}{c}{ Acidez Total Titulável $\left(\mathbf{M o l ~ L}^{-\mathbf{1}}\right)$} \\
\cline { 2 - 5 } & 0 dia & 4 dias & 8 dias & 12 dias \\
\hline Thiabendazole (Tecto $400 \mathrm{~mL} / 100 \mathrm{~L})$ & $2,11 \mathrm{bA}$ & $2,65 \mathrm{aB}$ & $0,68 \mathrm{cA}$ & $0,35 \mathrm{cA}$ \\
Prochloraz (Sportak $110 \mathrm{~mL} / 100 \mathrm{~L})$ & $2,11 \mathrm{bA}$ & $3,04 \mathrm{aB}$ & $0,73 \mathrm{cA}$ & $0,44 \mathrm{cA}$ \\
Imazalil (Magnate $200 \mathrm{~mL} / 100 \mathrm{~L})$ & $2,11 \mathrm{bA}$ & $3,01 \mathrm{aB}$ & $0,85 \mathrm{cA}$ & $0,40 \mathrm{cA}$ \\
Testemunha & $2,11 \mathrm{bA}$ & $3,66 \mathrm{aA}$ & $0,97 \mathrm{cA}$ & $0,40 \mathrm{dA}$ \\
\hline
\end{tabular}

Médias seguidas de mesma letra, minúscula na horizontal e maiúscula na vertical, não diferem entre si ao nível de 1\% de significância pelo Teste de Tukey. 
TABELA 7 - Valores médios de açúcares solúveis totais (\% de glicose, frutose e sacarose) de mangas cv. Van Dyke, após tratamento hidrotérmico e químico e armazenamento em temperatura ambiente $\left(25 \pm 2^{\circ} \mathrm{C}\right.$ e UR $\left.70 \%\right)$, por um período de 12 dias. Safra 2000, Janaúba - MG, ano 2000.

\begin{tabular}{clcccc}
\hline \multirow{2}{*}{ Banho } & \multicolumn{1}{c}{ Fungicidas } & \multicolumn{3}{c}{$\begin{array}{c}\text { Açúcares Solúveis Totais } \\
\text { (\% glicose, frutose e sacarose) }\end{array}$} \\
\cline { 3 - 6 } & & 0 dia & $\mathbf{4}$ dias & $\mathbf{8}$ dias & $\mathbf{1 2}$ dias \\
\hline \multirow{2}{*}{ Frio } & Thiabendazole (Tecto $400 \mathrm{~mL} / 100 \mathrm{~L})$ & $4,71 \mathrm{a}$ & $4,58 \mathrm{~b}$ & $10,56 \mathrm{~b}$ & $7,28 \mathrm{~b}$ \\
& Prochloraz (Sportak $110 \mathrm{~mL} / 100 \mathrm{~L})$ & $4,71 \mathrm{a}$ & $6,14 \mathrm{ab}$ & $11,95 \mathrm{ab}$ & $10,31 \mathrm{a}$ \\
& Imazalil (Magnate $200 \mathrm{~mL} / 100 \mathrm{~L})$ & $4,71 \mathrm{a}$ & $5,58 \mathrm{ab}$ & $13,41 \mathrm{a}$ & $9,17 \mathrm{a}$ \\
& Testemunha & $4,71 \mathrm{a}$ & $6,93 \mathrm{a}$ & $10,79 \mathrm{~b}$ & $8,75 \mathrm{ab}$ \\
\multirow{2}{*}{ Quente } & Thiabendazole (Tecto $400 \mathrm{~mL} / 100 \mathrm{~L})$ & $4,71 \mathrm{a}$ & $9,07 \mathrm{a}$ & $10,36 \mathrm{a}$ & $8,91 \mathrm{a}$ \\
& Prochloraz (Sportak $110 \mathrm{~mL} / 100 \mathrm{~L})$ & $4,71 \mathrm{a}$ & $5,08 \mathrm{~b}$ & $10,91 \mathrm{a}$ & $9,02 \mathrm{a}$ \\
& Imazalil (Magnate $200 \mathrm{~mL} / 100 \mathrm{~L})$ & $4,71 \mathrm{a}$ & $5,76 \mathrm{~b}$ & $10,41 \mathrm{a}$ & $8,98 \mathrm{a}$ \\
& Testemunha & $4,71 \mathrm{a}$ & $6,44 \mathrm{~b}$ & $11,45 \mathrm{a}$ & $9,70 \mathrm{a}$ \\
\hline
\end{tabular}

Médias seguidas de mesma letra, na vertical, não diferem entre si ao nível de $1 \%$ de significância pelo Teste de Tukey.

TABELA 8 - Porcentagem de infecção causada por Colletotrichum gloeosporioides em mangas cv. Van Dyke, após tratamento hidrotérmico e químico e armazenamento em temperatura ambiente $\left(25 \pm 2^{\circ} \mathrm{C}\right.$ e UR $\left.70 \%\right)$, por um período de 12 dias. Safra 2000, Janaúba - MG, ano 2000.

\begin{tabular}{lcccccc}
\hline \multirow{2}{*}{ Tratamentos } & \multicolumn{3}{c}{ Repetições } & \multicolumn{3}{c}{ Médias } \\
\cline { 2 - 7 } & I & II & III & IV & $\mathbf{1}$ & $\mathbf{2}$ \\
\hline Thiabendazole (Tecto $400 \mathrm{~mL} / 100 \mathrm{~L}$ ) & 24 & 12 & 56 & 28 & 30 & $5,39 \mathrm{abc}$ \\
Prochloraz (Sportak $110 \mathrm{~mL} / 100 \mathrm{~L}$ ) & 8 & 16 & 4 & 0 & 7 & $2,59 \mathrm{bc}$ \\
Imazalil (Magnate $200 \mathrm{~mL} / 100 \mathrm{~L}$ ) & 4 & 44 & 20 & 4 & 18 & $3,94 \mathrm{abc}$ \\
Thiabendazole (Tecto $400 \mathrm{~mL} / 100 \mathrm{~L}$ ) à $55^{\circ} \mathrm{C}$ & 4 & 24 & 4 & 24 & 14 & $3,62 \mathrm{abc}$ \\
Prochloraz (Sportak $110 \mathrm{~mL} / 100 \mathrm{~L}$ ) à $55^{\circ} \mathrm{C}$ & 0 & 0 & 0 & 0 & 0 & $1,0 \mathrm{c}$ \\
Imazalil (Magnate $200 \mathrm{~mL} / 100 \mathrm{~L}$ ) à $55^{\circ} \mathrm{C}$ & 4 & 40 & 0 & 0 & 11 & $2,66 \mathrm{bc}$ \\
Testemunha à $55^{\circ} \mathrm{C}$ & 28 & 56 & 24 & 44 & 38 & $6,16 \mathrm{ab}$ \\
Testemunha & 36 & 48 & 80 & 60 & 56 & $7,47 \mathrm{a}$ \\
\hline
\end{tabular}

1 - Média original; 2 - Média transformada por Raiz $(X+1)$. Médias seguidas de mesma letra, na vertical, não diferem entre si ao nível de $1 \%$ de significância pelo Teste de Tukey.

Ciênc. agrotec., Lavras, v. 29, n. 2, p. 289-295, mar./abr., 2005 


\section{CONCLUSÕES}

A associação do tratamento hidrotérmico e químico foi eficiente no controle da antracnose em frutos armazenados até 12 dias. O fungicida Prochloraz (Sportak $110 \mathrm{~mL} / 100 \mathrm{~L}$ ) associado ao tratamento hidrotérmico, inibiu totalmente o aparecimento de sintomas de antracnose.

\section{REFERÊNCIAS BIBLIOGRÁFICAS}

ASSOCIATION OF OFFICIAL ANALITICAL CHEMISTS. Official methods of analisys of the Association of Official Analitical Chemists. 15. ed. Washington, 1990. $2 \mathrm{v}$.

BLEINROTH, E. W. Frupex. In: NETTO, A. G. Manga para exportação: procedimentos de colheita e póscolheita. Brasília, DF: EMBRAPA-SPI, 1994. cap. 2, p. 11-28.

BLISKA, F. M. de M. Mercado interno de manga. In: SÃO JOSÉ, A. R. et al. Manga: tecnologia de produção e mercado. Vitória da Conquista, BA: DFZ/UESB, 1996. p. 241-252.

CHITARRA, M. I. F.; CHITARRA, A. B. Pós-colheita de frutos e hortaliças: fisiologia e manuseio. Lavras: ESAL/FAEPE, 1990. 320 p.

CUNHA, G. A. P. da et al. 6 variedades: cultivares. In: . Manga para exportação: aspectos técnicos da produção. Brasília, DF: EMBRAPA-SPI, 1994. p. 11-12.

GAYET, J. P. Frupex. In: NETTO, A. G. Manga para exportação: procedimentos de colheita e pós-colheita. Brasília, DF: EMBRAPA-SPI, 1994. p. 9-10.

GOMES, F. P. Curso de estatística experimental. 11. ed. São Paulo: Nobel, 1985. 466 p.

INSTITUTO ADOLFO LUTZ. Normas analíticas do Instituto Adolfo Lutz: métodos químicos e físicos para análise de alimentos. 3. ed. São Paulo, 1985. v. 1, 371 p.

KIST, H.; MANICA, I.; RECH, N. L. Características físico-químicas dos frutos de mangueiras cultivadas em região subtropical (Porto Lucena, RS). Revista Brasileira de Fruticultura, Cruz das Almas, v. 18, n. 3, p. 329-337, 1996.

MAIA, G. A. et al. Estudo da maturação de algumas variedades de manga (Mangifera indica L.). Revista Brasileira de Fruticultura, Cruz das Almas, v. 8, n. 2, p. 67-74, 1986.

MEDINA, V. M. Fisiologia e pós-colheita da manga. In: SÃO JOSÉ, A. R. et al. Manga: tecnologia de produção e mercado. Vitória da Conquista, BA: DFZ/UESB, 1996. p. 202-222.

NELSON, N. A. A photometric adaptation of Somoghy method for the determination of glucose. Journal of Biological Chemistry, Baltimore, v. 15, n. 1, p. 375-380, 1944.

RODRIGUES, J. A. S. Comportamento de dez variedades de manga (Mangifera indica $L$.) em Viçosa e Visconde do Rio Branco - Minas Gerais. Viçosa: UFV, 1977. 35 p.

SAMPAIO, J. M. M. Comportamento de cultivares de mangueira (Mangifera indica L.), em Cruz das Almas, Bahia: resultados preliminares. In: CONGRESSO BRASILEIRO DE FRUTICULTURA, 6., 1981, Recife. Anais... Recife: SBF, 1981. p. 943-951.

SÃO JOSÉ, A. R. Considerações gerais sobre a mangicultura. In: SÃO JOSÉ, A. R. et al. Manga: tecnologia de produção e mercado. Vitória da Conquista, BA: DFZ/UESB, 1996. p. 1-6.

SIQUEIRA, D. L. de et al. Características físicas e químicas de frutos de vinte cultivares de mangueira (Mangifera indica L.) em Uberaba MG. Revista Brasileira de Fruticultura, Cruz das Almas, v. 10, n. 2, p. 49-54, 1988.

SOUZA, S. J. F. de et al. Comparação de onze variedades de manga em Visconde do Rio Branco, Minas Gerais, visando ao consumo ao natural e á elaboração de geléias. Revista Ceres, Viçosa, v. 31, n. 178, p. 464478, 1984. 